cancer patients versus non-cancer controls across all phases of care (except terminal phase costs for cervical cancer). Outpatient costs were the major driver of costs across all post-diagnosis phases (figure 1 and 2).

Conclusion The mean phase-specific costs followed the following order: Terminal $>$ Initial $>$ Continuation $>$ Pre-diagnosis. This study highlights the substantial cost burden associated with cervical/endometrial cancer, and cost variation by phases of care.

Disclosures This study was funded by Merck Sharp \& Dohme Corp., a subsidiary of Merck \& Co., Inc., Kenilworth, NJ, USA. Chizoba Nwankwo is an employee of Merck Sharp \& Dohme Corp., a subsidiary of Merck \& Co., Inc., Kenilworth, NJ, USA. Anuj Shah, Ruchit Shah, Shelby Corman, and Nehemiah Kebede are employees of Pharmerit International, which received consulting fees related to this study.
385 SAFETY AND ANTITUMOR ACTIVITY OF DOSTARLIMAB IN PATIENTS (PTS) WITH ADVANCED OR RECURRENT DNA MISMATCH REPAIR DEFICIENT (DMMR) OR PROFICIENT (MMRP) ENDOMETRIAL CANCER (EC): RESULTS FROM THE GARNET STUDY

${ }^{1}$ Ana Oaknin, ${ }^{2}$ Lucy Gilbert, ${ }^{3}$ Anna V Tinker, ${ }^{4}$ Renaud Sabatier, ${ }^{5}$ Valentina Boni, ${ }^{6}$ David M O'malley, ${ }^{7}$ Sharad Ghamande, ${ }^{8}$ Wei Guo, ${ }^{8}$ Ellie Im, ${ }^{9}$ Bhavana Pothuri. ${ }^{1}$ Vall D'hebron University Hospital, Vall D'hebron Institute of Oncology (Vhio); ${ }^{2}$ Mcgill University Health Centre-Ri; ${ }^{3} B C$ Cancer; ${ }^{4}$ Institut Paoli Calmettes, Aix-Marseille University; Department of Medical Oncology; ${ }^{5}$ Centro Integral Oncológico Clara Campal, Hospital Universitario Hm Sanchinarro; ${ }^{6}$ The Ohio State University - James CCC; ${ }^{7}$ Georgia Cancer Center, Augusta University; ${ }^{8}$ Glaxosmithkline; ${ }^{9}$ New York University; Department of Obstetrics and Gynecology

\subsection{6/ijgc-2020-ESGO.71}

Introduction/Background Dostarlimab is a humanised programmed death (PD)-1 receptor monoclonal antibody that

Abstract 385 Table 1 Antitumour activity

\begin{tabular}{|c|c|c|}
\hline Variable & dMMR EC, $n=103$ & MMRp EC, n=142 \\
\hline Median follow-up time, mo & 16.3 & 11.5 \\
\hline $\begin{array}{l}\text { Objective response rate }{ }^{*} \text { n (\%, 95\% Cl) } \\
\text { Complete response, n (\%) } \\
\text { Partial response, n (\%) } \\
\text { Stable disease, n (\%) } \\
\text { Progressive disease, } \mathrm{n} \mathrm{( \% )} \\
\text { Not evaluable, } \mathrm{n}(\%) \\
\text { Not done, } \mathrm{n}(\%)\end{array}$ & $\begin{array}{c}46(\mathbf{4 4 . 7 \% , 3 4 . 9 - 5 4 . 8 )} \\
11(10.7) \\
35(34.0) \\
13(12.6) \\
39(37.9) \\
3(2.9) \\
2(1.9) \\
\end{array}$ & $\begin{array}{c}19(\mathbf{1 3 . 4 \% , 8 . 3 - 2 0 . 1 )} \\
3(2.1) \\
16(11.3) \\
31(21.8) \\
77(54.2) \\
0 \\
15(10.6) \\
\end{array}$ \\
\hline Disease control rate ${ }^{\dagger}, \mathrm{n}(\%, 95 \% \mathrm{Cl})$ & $59(57.3 \%, 47.2-67.0)$ & $50(35.2 \%, 27.4-43.7)$ \\
\hline Response ongoing, $\mathbf{n}(\%)$ & $41(89.1)$ & $12(63.2)$ \\
\hline Median duration of response, (range) mo & Not reached (2.63-28.09+) & Not reached $(1.54+-30.36+)$ \\
\hline $\begin{array}{l}\text { Kaplan-Meier estimated probability of } \\
\text { remaining in response } \\
\text { at } 6 \mathrm{mo}, \% \\
\text { at } 12 \mathrm{mo}, \% \\
\text { at } 18 \mathrm{mo}, \%\end{array}$ & $\begin{array}{l}97.8 \\
90.6 \\
79.2 \\
\end{array}$ & $\begin{array}{l}83.0 \\
61.3 \\
61.3 \\
\end{array}$ \\
\hline
\end{tabular}

*Responses required confirmation at a subsequent scan; SD had to be observed at $\geq 12$ weeks on study to qualify as SD; †Includes confirmed CR, PR or SD at $\geq 12$ weeks.

$\mathrm{CR}$, complete response; $\mathrm{dMMR}$, mismatch mutation repair deficient; $\mathrm{EC}$, endometrial cancer; MMRp, mismatch mutation repair proficient, ORR, objective response rate; $P R$, partial response; SD, stable disease.

Abstract 385 Table 2 Most common adverse events

\begin{tabular}{|c|c|c|c|}
\hline MedDRA preferred term, $\mathrm{n}(\%)$ & $\begin{array}{c}\text { dMMR EC } \\
\text { N=126 }\end{array}$ & $\begin{array}{c}\text { MMRp EC } \\
\mathrm{N}=145\end{array}$ & $\begin{array}{l}\text { Overall } \\
\mathrm{N}=271\end{array}$ \\
\hline \multicolumn{4}{|l|}{ Any grade TRAEs } \\
\hline Fatigue & $17(13.5)$ & $30(20.7)$ & $47(17.3)$ \\
\hline Diarrhoea & $20(15.9)$ & $19(13.1)$ & $39(14.4)$ \\
\hline Nausea & $16(12.7)$ & $21(14.5)$ & $37(13.7)$ \\
\hline \multicolumn{4}{|l|}{ Grade $\geq 3$ TRAEs } \\
\hline Anaemia & $5(4.0)$ & $2(1.4)$ & $7(2.6)$ \\
\hline $\begin{array}{l}\text { Alanine aminotransferase } \\
\text { increased }\end{array}$ & $2(1.6)$ & $2(1.4)$ & $4(1.5)$ \\
\hline Diarrhoea & $2(1.6)$ & $2(1.4)$ & $4(1.5)$ \\
\hline \multicolumn{4}{|l|}{ TRAEs leading to discontinuation } \\
\hline $\begin{array}{l}\text { Alanine aminotransferase } \\
\text { increased }\end{array}$ & $1(0.8)$ & $2(1.4)$ & $3(1.1)$ \\
\hline $\begin{array}{l}\text { Aspartate aminotransferase } \\
\text { increased }\end{array}$ & $1(0.8)$ & $1(0.7)$ & $2(0.7)$ \\
\hline Transaminases increased & $2(1.6)$ & 0 & $2(0.7)$ \\
\hline
\end{tabular}

dMMR, mismatch mutation repair deficient; EC, endometrial cancer; MMRp, mismatch mutation repair proficient; TRAE, treatment-related adverse event. 
blocks interaction with the PD-1 ligands, PD-L1 and -L2. GARNET is a phase 1 study assessing antitumour activity and safety of dostarlimab monotherapy in patients with advanced solid tumours.

Methodology This multicentre, open-label, single-arm study is being conducted in 2 parts, dose escalation and expansion. Here we report on 2 independent expansion cohorts of patients with recurrent or advanced endometrial cancer (EC) that progressed on or after a platinum-based chemotherapy regimen. Assignment to cohort A1 (mismatch mutation repair deficient [dMMR] EC) or cohort A2 (mismatch mutation repair proficient [MMRp] EC) was determined by immunohistochemistry (IHC) testing. Patients received $500 \mathrm{mg}$ dostarlimab intravenously once every 3 weeks for 4 cycles, then $1000 \mathrm{mg}$ once every 6 weeks until disease progression, discontinuation or withdrawal. The primary endpoints are objective response rate (ORR) and duration of response (DOR) by blinded independent central review using RECIST version 1.1.

Results In total, $126 \mathrm{dMMR}$ and 145 MMRp pts identified by IHC were enrolled and dosed. Of these, $103 \mathrm{dMMR}$ and 142 MMRp pts had measurable disease as baseline and sufficient follow-up time (6 months) for efficacy analyses, respectively. Patients that progressed prior to 6 months were included in the evaluable population. ORR for dMMR EC was 44.7\%; ORR for MMRp EC was $13.4 \%$ (table 1). Median DOR and OS were not reached in either cohort. Overall, 15 pts (5.5\%) discontinued treatment due a TRAE (5 dMMR, 10 MMRp). Safety by cohort and overall are shown in table 2. There were no deaths attributed to dostarlimab.

Conclusion Dostarlimab demonstrated durable antitumour activity in both $\mathrm{dMMR}$ and MMRp advanced/recurrent EC. dMMR status by IHC was associated with a higher response rate. Dostarlimab demonstrated a notable disease control rate $(35.2 \% ; 2.1 \%$ complete response, $11.3 \%$ partial response, $21.8 \%$ stable disease) in patients with MMRp EC, which comprised a higher percentage of patients with Type II EC and is historically associated with a worse prognosis. No new safety signals were detected. These cohorts are the largest prospective evaluation of a PD-(L)1 therapy in EC to date.

Disclosures Clinical trial registration: NCT02715284

This study was sponsored by GlaxoSmithKline, Waltham, MA, USA.

Dr. Oaknin reports consulting and honoraria from AstraZeneca, Tesaro, Clovis, PharmaMar, and Roche.

Dr. Gilbert reports honoraria from Meck, AstraZeneca, and Pfizer.

Dr. Tinker reports grants and personal fees from AstraZeneca.

Dr. Sabatier reports grants from EISAI and AstraZeneca; personal fees from Roche, Pfizer, Tesaro, Novartis and AstraZeneca; and non-financial support from Roche, Pfizer, AstraZeneca, and Amgen.

Dr. O’Malley reports personal fees from Immunogen, Eisai, Agenus, GSK : Consultant/Advisory Board for Clovis, Ambry, Abbvie, Janssen/J\&J, Regeneron, Novacure, Myraid Genetics, Tarveda, Amgen, VentiRx, Array Biopharma, EMD Serono, Ergomed; Steering committee for Genentech/Roche and Merck; Institutional funding from Ajinomoto Inc, Ludwig Cancer Research, Stemcentrx, Inc, CERULEAN PHARMA, GOG Foundation, BMS, Serono Inc, TRACON Pharmaceuticals, Yale University, New Mexico Cancer Care Alliance, INC Research, Inc., Inventiv Health Clinical, Iovance Biotherapeutics, Inc, and PRA International.
Dr. Ghamande reports consulting fees from Seattle Genetics; speakers' bureau fees from GSK; and institutional grants from GSK, Merck, Roche, Genentech, Takeda, Seattle Genetics, Advaxis, BMS, Clovis, Abbvie, and Tesaro.

Dr. Pothuri reports grants, personal fees and non-financial support from GSK; Advisory Board fees from AstraZeneca and Clovis Oncology.

Dr. Boni has nothing to disclose.

Drs. Guo and Im are employees of GlaxoSmithKline.

\section{PATIENT-REPORTED OUTCOMES (PROS) IN THE GARNET TRIAL IN PATIENTS (PTS) WITH ADVANCED OR RECURRENT MISMATCH REPAIR DEFICIENT/ MICROSATELITE INSTABILITY-HIGH (DMMR/MSI-H) ENDOMETRIAL CANCER (EC) TREATED WITH DOSTARLIMAB}

${ }^{1}$ Rebecca Kristeleit, ${ }^{2}$ Cara Mathews, ${ }^{3}$ Andrés Redondo, ${ }^{4}$ Joice Huang, ${ }^{4}$ Laurie Eliason, ${ }^{4}$ Ellie Im, ${ }^{5}$ Jubilee Brown. ${ }^{1}$ Guy's and St. Thomas Hospitals, NHS Foundation Trust; ${ }^{2}$ Women and Infants Hospital of Rhode Island; ${ }^{3}$ Hospital Universitario La Paz - Idipaz; ${ }^{4}$ Glaxosmithkline; ${ }^{5}$ Levine Institute, Atrium Health

\subsection{6/ijgc-2020-ESG0.72}

Introduction/Background PROs enable direct measurement of the experiences of pts with cancer related to an intervention. Regulators increasingly use PROs to inform the risks and benefits of new drug candidates, focusing on 3 core concepts: physical functioning (PF), disease-related symptoms (DRS), and symptomatic adverse events (AEs).

Dostarlimab is an investigational anti-programmed death-1 monoclonal antibody that has shown activity in pts with advanced dMMR EC (objective response rate, 42\%; disease control rate, 58\%) and an acceptable safety profile. Here, we report on PROs in pts treated with dostarlimab in the singlearm GARNET trial.

Methodology Pts with recurrent or advanced dMMR/MSI-H EC that progressed on a platinum regimen received $500 \mathrm{mg}$ Q3W*4 of dostarlimab, then $1000 \mathrm{mg}$ Q6W until disease progression or discontinuation (DC). PRO assessment, an exploratory endpoint, was measured using the EORTC-QLQ-C30. PROs were collected at baseline (BL), each dose cycle, and after DC. For PF and DRS (pain and fatigue), we conducted multi-item descriptive analyses, including change from BL. For symptomatic AEs and tolerability (nausea, vomiting, constipation, diarrhoea, tiredness/fatigue), we conducted item-level analyses to understand response distribution and change in response categories from BL: improved, stable, and 1-, 2-, or 3 -category worsening.

Results PRO data were available for 66/104 pts who received $\geq 1$ dose of dostarlimab. Questionnaire compliance was consistent across domains, ranging from $100 \%$ at $\mathrm{BL}$ to $45 \%$ at cycle 7. Pain, fatigue, and PF were maintained above BL starting at cycles 1,3 , and 4, respectively. Symptomatic AEs were experienced by a minority of pts, with $<25 \%$ and $<6 \%$ of pts having 1- or $\geq 2$-category worsening, respectively. Improved scores were reported by $6 \%$ to $37 \%$ of pts.

Conclusions PROs from the GARNET trial showed that dostarlimab was generally well tolerated and disease-related symptoms were improved or maintained while on treatment. These data, along with the efficacy and safety profile of dostarlimab, support use of dostarlimab in pts with dMMR/MSI-H advanced EC.

Disclosures Clinical trial registration: NCT02715284 